\title{
A mathematical model of force on the control column of a low-cost flight simulator
} R G Davies ${ }^{1} \quad$ G Sen Gupta ${ }^{2}$

Received 17 November 2017; revised 23 April 2018

\begin{abstract}
The personal computer based aviation training device (PCATD) is a type of low cost flight simulator which is widely used in the general aviation industry in procedural flight training. Apart from visual effects, a PCATD does not provide trainee pilots with the same physical experience as the physical feeling they receive in actual flights. To improve the fidelity in flight control of PCATD for our Diamond DA-40 trainer aircraft, a force feed-back system is proposed for one of the flight control systems, the control column in the cockpit. The core element of this proposed system is the mathematical model to simulate the resultant forces on the control column when a manoeuvre is performed. This mathematical model considers the general aerodynamic forces exerted on the control surfaces, and the specific aerodynamic forces caused by the configuration of the aircraft, the static forces and dynamic
\end{abstract}

DOI:10.21914/anziamj.v59i0.12659, (c) Austral. Mathematical Soc. 2018. Published November 9, 2018, as part of the Proceedings of the 13th Biennial Engineering Mathematics and Applications Conference. ISSN 1445-8810. (Print two pages per sheet of paper.) Copies of this article must not be made otherwise available on the internet; instead link directly to the DOI for this article. 
forces along the control transmission systems. This model is two dimensional only, pitching and rolling, which are performed by the control column. A special regime of detecting the movement of the control column with visual analysis is developed due to the dimensional and regulatory restrictions in a cockpit. The force feed-back on the control column following the movement of the control is calculated by the model. The results of the calculations explain the features of the components of the total force on control, which will assist the planning and implementation of applying this force model in the simulator.

Subject class: $34 ; 39$

Keywords: force; flight simulator

\section{Contents}

1 Introduction

C233

2 Mechanics of a flight control

C235

3 Mathematical model of force on control C236

3.1 Aerodynamic force . . . . . . . . . . . . . C237

3.2 Inertia force . . . . . . . . . . . . . . . . . C C239

3.3 Frictional force . . . . . . . . . . . . . . . . . C241

3.4 Spring force . . . . . . . . . . . . . . . C243

3.5 The total force on control column . . . . . . . . . . . C243

4 Measurement of the movement of control column

5 Model calculation

6 Conclusion 


\section{Introduction}

A flight simulator used for procedural practice in general aviation usually is a personal computer based aviation training device (PCATD) (General aviation is the term used for civil aviation operations other than scheduled or unscheduled air services for passengers or freight). A PCATD type simulator looks like the interior of a real cockpit with a screen displaying the view as a pilot can see it in flight. However, there is no real feel of heaviness on the control and the movement of the "cockpit" when the operator, who can be a trainee pilot, moves the control column, or control yoke to perform "manoeuvres". The lack of feel of the motion in the cockpit can be compensated by the moving scenery displayed on the screen, but the lack of a realistic feeling of the heaviness of the control is a main short-coming for a PCATD.

Full-Flight-Simulators can simulate the full motion and full force feed-back on the control, and the operator in one of these simulators should have the same experience as in a real flight in air. The Full-Flight-Simulators are equipped with hydraulic power systems and complex control networks. For example, a complex model was built by Nam [4] to describe only a small portion of a control network for one of this type of simulator. A Full-Flight-Simulator bears high cost, and usually only organisations like commercial airlines can afford to use them.

To develop a relatively simple system with force feed-back onto the control column in a low-cost simulator is strategically important for future flight training in general aviation, because it can provide trainee pilots with a real sense of force on the control column, and it potentially can decrease the number of hours for flight training, and increase the number of hours in the simulator. A hour of flight training costs several hundred dollars, while a hour of simulator training costs a fraction of that of flight training, so the training cost can be decreased. Trainee pilots can be better prepared in the simulator, and reduce operational errors made in flight, so less possibility of having training accidents, and less insurance claims are required, which is 
another cost saving. And, it would modernise the training process in general aviation. However, there are very few simulators with force feed-back features used in general aviation, and information on the force model for the force feed-back to the control of any simulator for flight training is limited in the public domain.

In the automotive industry, some low cost simulators have been developed, which can have the main functions of a fully equipped simulator, but are more affordable, and relatively easy to use [5]. This principle should be readily workable for flight simulators.

The basis of applying the correct force to a control column is a well-thought mathematical model, which can describe the actions of flight control surfaces, and the forces caused by flight manoeuvres. The flight control surfaces are elevator, ailerons and rudder. Flight manoeuvres are performed by operating the control column, also known as the "control". Then the model is tested and analysed using the actual movement of a control column in a real aeroplane. The final stage of implementation of the force feed-back feature into a PCATD simulator is to select an actuating system to apply the force calculated by the model with the flight data from the simulator and the movement of the control column of the simulator.

Usually a flight simulator is specifically built for a particular model of aeroplane. For example, there are three models in Diamond series of flight trainers: DA-40, DA-42, and DA-62, which require three different simulators for flight training. So, our model development also considers the modelling of the key elements in the transmission systems in individual modules, which in the future can be tailored to suit the requirements of different models of aeroplanes. For example, one force feed-back system could then be used for DA-40, DA-42, and DA-62.

The aeroplane used in this work is one of the Diamond single engine trainers DA-40 at the School of Aviation, Massey University, and the simulator, with which the force model will be implemented, is an in-house built PCATD [3]. 
The work involved in this paper is the mathematical model of forces on the control of DA-40, the measurement strategy to obtain the real movement of control in the aeroplane, and the force calculations by the model following the measured movement of a control column. The measurement strategy for the movement of the control column in the cockpit has to overcome the restrictions practised in aviation, in particular in flight.

\section{Mechanics of a flight control}

The flight control system of a general aviation aircraft is usually a mechanical system, which is simple and reliable. An elevator is a part of a tail plane, which controls the pitching motion of the aeroplane. Ailerons are situated at the trailing edge of wings, and control the rolling motion of the plane. A flight control system, for example, the control system for elevator as shown in Figure 1, consists of control column (or yoke), in the cockpit, rotating arms, rods, movement restriction springs, and the elevator. The rotating arms and rods transmit the motion from the control in cockpit to the flight control surface i.e. elevator.

In the cockpit of a Diamond DC-40, a pilot operates the control surfaces, elevator and ailerons by moving the control column (the stick). When the pilot wants to climb, the control column is pulled back, and the control column rotates about a pivot on the column, and the other end of the control column drives a longitudinal transmission rod forward. This rod is more than $5 \mathrm{~m}$ long sitting on five supporting seats. This longitudinal rod is jointed with the second rod, the vertical control rod in the tail section of the plane by a rotating arm, and pulls this vertical control rod downward. The other end of the vertical rod is connected with the elevator by another rotating arm to deflect the elevator with a negative deflection angle, so the elevator will produce the aerodynamic force, which will provide the plane with a nose-up pitch moment. The plane will climb. 


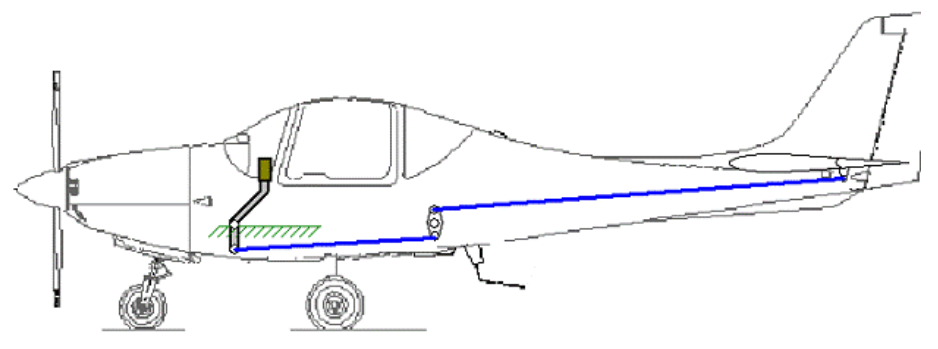

Figure 1: Elevator linkage

The function of the rotating arms is to connect two moving parts and change the direction of the movements. The supporting seats provide the guide of motion to the longitudinal rod and prevent the rod from sagging. However, there will be friction between the rod and a seat, when the rod is moving. The range of the deflection of the elevator is restricted, because the elevator can stall if the deflection angle exceeds approximately $15^{\circ}$. Springs are installed along the second rod to prevent the elevator from deflecting up or down excessively.

The aileron control system is very similar to the elevator control system in terms of the operational principle of the mechanics, although the number and size of the components of transmission rods, rotating arms, and the restricting springs are different.

The mathematical model of the force to the control column is built on the basis of the mechanics of the control system.

\section{Mathematical model of force on control}

The mathematical model should describe the force to the pilot's hand, when the pilot moves the control column in flight. The pilot's hand has to overcome 
the aerodynamic forces produced on the control surfaces, i.e. ailerons and elevator, the inertia from the the linkage rods and rotating arms, the resistance generated by the springs in the control systems and the friction between the moving parts and the surfaces of their supports. Additionally there is an aerodynamic force caused by the slipstream from the propeller of the aeroplane on the flight control surface. However, the effect of this force on the control surface depends on the configuration of the powerplant of the aeroplane, i.e. single engine or twin engine is a constant component on the flight control surfaces. When the plane is in flight, it can be trimmed off by a trim tab on the flight control surface. Therefore, it is neglected in this model. It will be considered in a more advanced model.

This model is 2-dimensional, longitudinal and lateral. The directions of the force on the control column are defined as:

- The direction of the force acting on the control column, when it moves forward/backward to deflect the elevator, is Longitudinal, and the displacement of the control column in this direction is $\mathbf{y}$; Moving forward is " + ".

- The direction of the force when the control column moves sideways, right/left, is Lateral, and the displacement of the control column in this direction is $x$; moving right is " + ".

The mathematical description of these forces will be explained in the following subsections.

\subsection{Aerodynamic force}

When a flight control surface deflects, it rotates about its hinge by an angle $\delta$, the deflection angle, as shown in Figure 2. The flight control surface produces lift, which will generate a moment to control the plane into an expected action. 


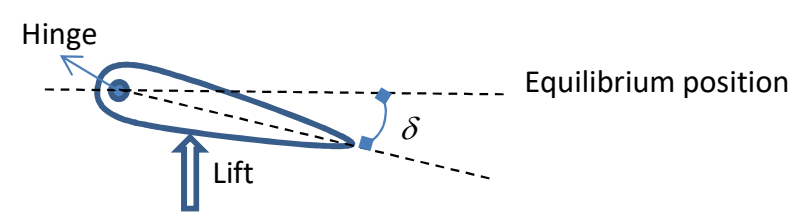

Figure 2: Flight control surface

Longitudinal: When elevator deflects from its equilibrium position in flight with a deflection angle $\delta_{e}$, lift will be produced on this surface. This lift, $\mathrm{L}_{e}$ is expressed as [2]

$$
\mathrm{L}_{e}=\mathrm{C}_{\mathrm{Le}} \frac{1}{2} \rho v^{2} \mathrm{~S}_{e},
$$

where $\rho$ is density of air; $v$ is airspeed; $S_{e}$ is the area of elevator. $C_{L e}$ is lift coefficient of elevator. An elevator is assumed as a symmetrical thin aerofoil. According to the formula to determine the lift coefficient of a symmetrical thin aerofoil [1], the lift coefficient of the elevator $\mathrm{C}_{\mathrm{Le}}$ is calculated by

$$
\mathrm{C}_{\mathrm{Le}}=2 \pi \delta_{e},
$$

where $\delta_{e}$ is elevator's deflection angle.

This lift $\mathrm{L}_{e}$ will be transmitted to the control column in the cockpit through the elevator control linkage as $F_{A y}$,

$$
F_{A y}=\beta_{e} L_{e}=\beta_{e} C_{L e} \frac{1}{2} \rho v^{2} S_{e},
$$

where $\beta_{e}$ is the transmission coefficient determined by the ratios of the radii of the rotating arms in the elevator linkage system.

Lateral: When ailerons deflect from their equilibrium position in flight with a deflection angle $\delta_{\mathrm{a}}$, lift will be produced, and will make the aeroplane roll. The ailerons on the two wings deflect at the same time in opposite directions as shown in Figure 3. If the force transmitted to the control column is "pull" from left aileron, the force from the right aileron will be "push". But to the 
hand of pilot on the control column, as shown in Figure 4, they always are in the same direction.

The lift produced by an aileron, when it deflects, is

$$
\mathrm{L}_{\mathrm{a}}=\mathrm{C}_{\mathrm{La}} \frac{1}{2} \rho v^{2} \mathrm{~S}_{\mathrm{a}},
$$

where $\rho$ is density of air; $v$ is airspeed; $S_{a}$ is the area of one aileron. $C_{L a}$ is lift coefficient of elevator. As for the elevator's lift coefficient, the lift coefficient of aileron $\mathrm{C}_{\mathrm{La}}$ is calculated by

$$
\mathrm{C}_{\mathrm{La}}=2 \pi \delta_{\mathrm{a}},
$$

where $\delta_{a}$ is elevator's deflection angle.

The $L_{a}$ by the two ailerons will be transmitted to the control column in the cockpit though the aileron control linkage as $F_{A x}$,

$$
F_{A x}=\beta_{\mathrm{a}} 2 L_{e}=\beta_{\mathrm{a}} 2 \mathrm{C}_{\mathrm{La}} \frac{1}{2} \rho v^{2} \mathrm{~S}_{\mathrm{a}},
$$

where $\beta_{\mathrm{a}}$ is the transmission coefficient determined by the ratios of the radii of the rotating arms in the aileron linkage system.

\subsection{Inertia force}

The pilot's hand has to overcome the inertia of the control linkage systems to initiate an acceleration, so the linkage system can move and act on the flight control surfaces. The rods in the linkage systems perform translational movement, while the rotating arms are in rotational movement. The rods require forces to produce translational acceleration, and the arms require forces to generate moments or torque to produce angular acceleration.

The rods are uniform cylindrical rods. A rotating arm is treated as a short cylindrical rod pivoting about one end. The total inertia forces are: 


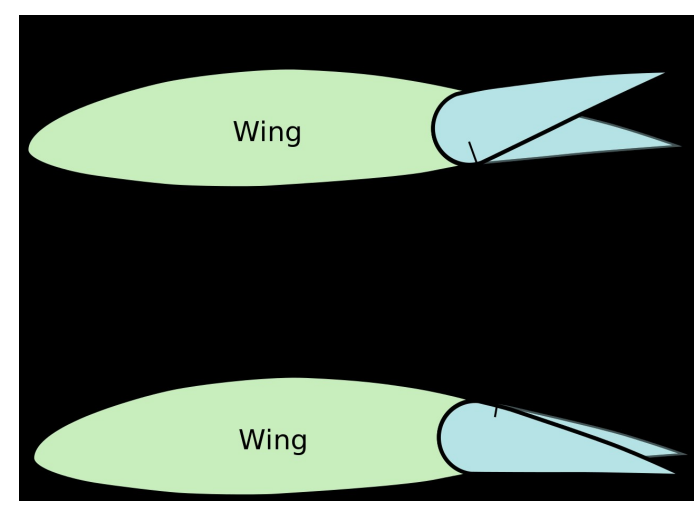

Figure 3: Ailerons

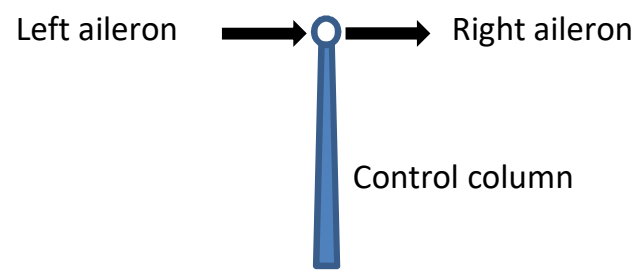

Figure 4: Force of aileron on control column

Longitudinal:

$$
F_{I y}=\sum_{i=1}^{N_{r}} m_{i} \frac{d^{2} y_{i}}{d t^{2}}+\sum_{j=1}^{N_{a r}} \frac{I_{j}}{r_{j}} \frac{d^{2} \theta_{j}}{d t^{2}},
$$

where

$r_{j}$ and $I_{j}$ are the length and the moment of inertia of the $j$ th rotating arm, and $I_{j}=\frac{m_{j}}{3} r_{j}^{2}$;

$\theta_{j}$ is the angular displacement of the rotating arm, and $\theta_{j} \approx \frac{y_{j}}{r_{j}}$, since $\theta_{j}$ is relatively small;

$t$ is time; 
$\mathrm{N}_{\mathrm{r}}$ and $\mathrm{N}_{\mathrm{ar}}$ are the number of rods and the number of rotating arms in the elevator control linkage respectively;

$m_{i}$ and $m_{j}$ are the mass of the $i$ rod and $j$ th arm respectively;

$y_{i}, y_{j}$ are the displacement of $i$ th rod and the moving end of the $j$ arm respectively, and $y_{i}=\kappa_{i} y, y_{j}=\kappa_{j} y ; \kappa_{i}$ and $\kappa_{j}$ are the transmission ratios from the control column to the $i$ th rod and $j$ th arm.

\section{Lateral:}

$$
F_{I x}=\sum_{k=1}^{M_{r}} m_{k} \frac{d^{2} \chi_{k}}{d t^{2}}+\sum_{l=1}^{M_{a r}} \frac{I_{l}}{r_{l}} \frac{d^{2} \phi_{l}}{d t^{2}}
$$

where

$r_{l}$ and $I_{l}$ are the length and the moment of inertia of the lth rotating arm, and $\mathrm{I}_{l}=\frac{\mathrm{m}_{l}}{3} \mathrm{r}_{l}^{2}$;

$\phi_{\mathrm{l}}$ is the angular displacement of the lth rotating arm, and $\phi_{\mathrm{l}} \approx \frac{\mathrm{x}_{\mathrm{l}}}{\mathrm{r}_{\mathrm{l}}}$, since $\phi_{l}$ is relatively small;

$t$ is time;

$M_{r}$ and $M_{a r}$ are the number of rods and the number of rotating arms in the aileron control linkage respectively;

$m_{k}$ and $m_{l}$ are the mass of the $k$ th rod and $l$ th arm respectively;

$x_{k}, x_{l}$ are the displacement of $k$ th rod and the moving end of the lth arm respectively, and $x_{k}=\kappa_{k} x, x_{l}=\kappa_{l} x ; \kappa_{k}$ and $\kappa_{l}$ are the transmission ratios from the control column to the ith rod and $j$ th arm.

\subsection{Frictional force}

There are long rods in the linkage systems, in particular, in the elevator control linkage. A long rod of the linkage usually passes through one or two support or guiding holes (seats) either longitudinally, or vertically. However, 
there are no seats for the shorter rods. A long rod moves against the surface of the holes when it is in operation. There is friction between the rod and the holes.

There is friction at the pivot of a rotating arm, so this frictional force will be considered at every rotating arm.

It is assumed that the friction acts on the surface of the moving parts similar to drag on a surface. The drag is proportional to the dynamic force on the surface, which is assumed to be proportional to the magnitude of acceleration of the moving parts. The greater the acceleration is, the harder the moving part is pressed on the adjacent stationary surface, and the higher the dynamic force on the surface. The expression of frictional forces in the model is similar to inertial forces of their corresponding parts, and the direction of friction is always opposite to the velocity of the moving parts. The force from the pilot's hand to overcome the friction is in the same direction as that of the velocity of the moving parts. The expressions of frictional forces are

\section{Longitudinal:}

$$
F_{F y}=\sum_{i=1}^{N_{s t}} \mu_{i} m_{i} \frac{\frac{d y_{i}}{d t}}{\left|\frac{d y_{i}}{d t}\right|}\left|\frac{d^{2} y_{i}}{d t^{2}}\right|+\sum_{j=1}^{N_{a r}} \mu_{j} \frac{I_{j}}{r_{j}} \frac{\frac{d \theta_{j}}{d t}}{\left|\frac{d \theta_{j}}{d t}\right|}\left|\frac{d^{2} \theta_{j}}{d t^{2}}\right|+\mu_{a} F_{A y}
$$

where $\mathbf{N}_{s t}$ is number of supporting seats in the elevator linkage system, and $\mu_{i}$ and $\mu_{j}$ are the friction coefficients, which depend on the quality of the contacting surfaces and types of relative motion. $\mu_{\mathrm{a}}$ is the friction coefficient due to aerodynamic forces. Other parameters are the same as those used in Section 3.2.

\section{Lateral:}

$$
F_{F x}=\sum_{i=k}^{M_{s t}} \mu_{k} m_{k} \frac{\frac{d x_{k}}{d t}}{\left|\frac{d x_{k}}{d t}\right|}\left|\frac{d^{2} x_{k}}{d t^{2}}\right|+\sum_{l=1}^{M_{a r}} \mu_{l} \frac{I_{l}}{r_{l}} \frac{\frac{d \theta_{l}}{d t}}{\left|\frac{d \theta_{l}}{d t}\right|}\left|\frac{d^{2} \theta_{l}}{d t^{2}}\right|,+\mu_{a} F_{A x},
$$

where $M_{s t}$ is number of supporting seats in the aileron linkage system, and $\mu_{k}$ and $\mu_{l}$ are the friction coefficients. $\mu_{a}$ is the friction coefficient due 
to aerodynamic forces. Other parameters are the same as those used in section 3.2.

\subsection{Spring force}

The forces produced by the restriction springs are always in the opposite direction of their displacements using Hooke's Law. But the force from the pilot's hand to overcome the spring force is in the same direction as the displacement. So the forces caused by the restriction springs are

\section{Longitudinal:}

$$
F_{S y}=\sum_{i=1}^{N_{s e}} \lambda_{i} y_{i},
$$

where $\mathrm{N}_{\mathrm{se}}$ is the number of restriction springs in elevator control linkage system; $\lambda_{i}$ and $y_{i}$ are the spring constant and the displacement of the $i$ th spring respectively; and $y_{i}=\kappa_{s i} y$, and $\kappa_{s i}$ is the transmission ratio from the control column to this spring.

\section{Lateral:}

$$
F_{S x}=\sum_{j=1}^{N_{s a}} \lambda_{j} x_{j},
$$

where $\mathrm{N}_{\mathrm{sa}}$ is the number of restriction springs in aileron control linkage system; $\lambda_{j}$ and $x_{j}$ are the spring constant and the displacement of the $j$ th spring in the aileron system respectively; and $x_{j}=\kappa_{s j} x$, and $\kappa_{s j}$ is the transmission ratio from the control column to this spring.

\subsection{The total force on control column}

The complete model describes the total force exerted on the control column in cockpit. The total force in longitudinal direction is 


$$
F_{y}=F_{A y}+F_{F y}+F_{F y}+F_{S y},
$$

determined by equations (3), (7),(9), and (11).

The total force force in longitudinal direction is

$$
F_{x}=F_{A x}+F_{F x}+F_{F x}+F_{S x},
$$

determined by equations (6), (8),(10), and (12).

The direction of the total force is determined by

$$
\gamma=\arctan \left(\frac{F_{y}}{F_{x}}\right)
$$

\section{Measurement of the movement of control column}

The method of measuring the movement of the control column is under various restrictions due to stringent aviation rules. Moreover, the space available in the cockpit is also limited.

In order to measure the movement of the control column, a vision based approach was adopted. A camera, connected to a Raspberry Pi single-board computer (SBC), was mounted on the cockpit canopy looking downwards at the control column. A coloured sticker was attached to the top of the control column and it was tracked by the image processing software. Images taken by the camera are processed by the SBC and the coordinates of the coloured sticker are calculated. These coordinates are time stamped and sent to a laptop over a serial communication link. The transmitted coordinates are stored in a file in the laptop for post processing. From the position data collected, it is also possible to calculate the velocity of the control column 


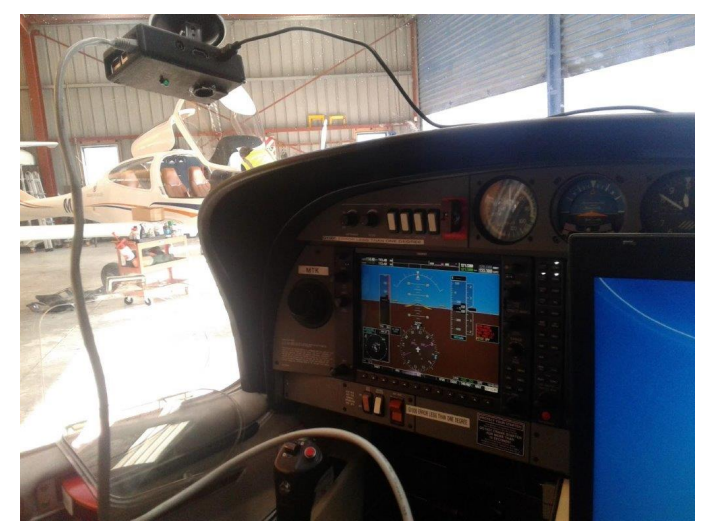

Figure 5: Camera mounted on the cockpit canopy of Diamond DA-40

movements in a two-dimensional plane. The camera, when mounted, left a relatively small vertical gap of $38.5 \mathrm{~cm}$ between the lens and the sticker at the top of the control column. A wide-angle lens was thus used to cover a wider field of view and ensure that the sticker is still visible when the control column is in its extreme positions. The mounted camera on the cockpit canopy is shown in Figure 5.

Tracking only one coloured object in an image frame is fast and is comfortably handled by the processing power of the Raspberry Pi. The image processing software was written in Python and OpenCV library routines were used. The various stages of image processing are shown in Figure 6. The output of this process described by this flow chart is the displacement and the velocity of the control column.

\section{Model calculation}

With the method discussed in Section 4, the measurement of the control column in the cockpit of one DA-40 can be detected and coordinates of the 


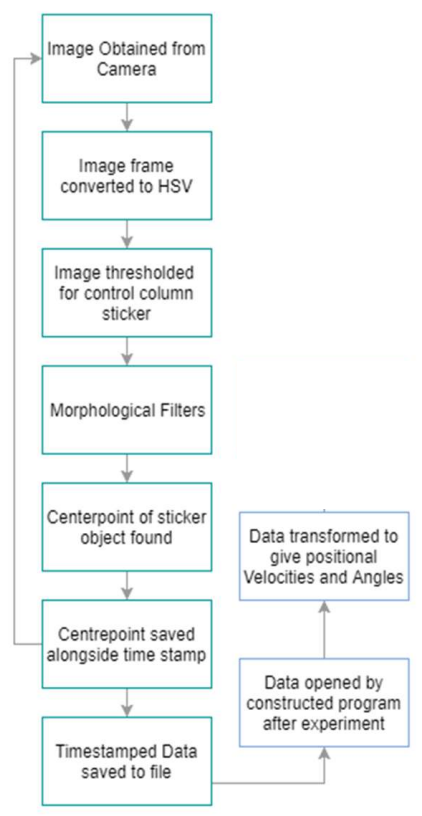

Figure 6: Flow diagram of image processing program running on SBC

control column in longitudinal- $y$ direction, and lateral- $x$ direction can be traced and recorded as time series $y(t)$ and $x(t)$.

One of the measurements of control column's movement is shown in Figure 7. The instructions for the action in this Figure were to move the control column laterally to the right (to operate ailerons), in $x$ direction, and then move back to the neutral position. However, there still is a slight movement in $y$ which has been detected. The accelerations in these two directions are also displayed in this Figure. The component of the acceleration in $y$ direction is small, so the action is treated as a lateral movement. Two dimensional calculations were carried out, and the components of the forces in longitudinal (y) were very weak, so the discussion on the results concentrates on the lateral movement. 
The lateral movement means that the control column operates the ailerons. In the aileron linkage system, there are 7 rotating arms and 4 rods to transmit the action from the control in the cockpit to each aileron. The force calculation below is based on this aileron linkage system.

The equations of the force model indicates that inertia and frictional forces are proportional to the the accelerations $\frac{\mathrm{d}^{2} x}{\mathrm{dt}^{2}}$ and $\frac{\mathrm{d}^{2} \theta}{\mathrm{dt}^{2}}$, and The spring forces are proportional to the displacement $x(t)$, while the aerodynamic force is proportional to the deflection angle $\delta$ if the airspeed is constant.

Force calculation scenario 1: on ground

When the aeroplane parks on ground (engine off), there is no aerodynamic force exerted on the flight control surface. The force on the control column following the movement shown in Figure 7 is calculated. The components of the calculated force in $x$ direction together with the lateral $(x)$ movement of the control column are shown in Figure 8.

It is clear that the spring component of the force, labeled as $\mathrm{F}_{-} \mathrm{Sx}$, follows the profile of the displacement, and the component of inertia and friction, labeled as F_Ix, follows the profile of the acceleration of the control column. The total force on the control on ground is the force caused by the mechanics of the control surface linkage system, which will be known as the system force in the following context.

Force calculation scenario 2: in flight

The airspeed required in the model should be obtained from the flight data of the aeroplane. Since the testing flight has not been able to be carried out, here the total forces on the control column are calculated at two constant airspeeds: $60 \mathrm{kt}$ and $110 \mathrm{kt}$, following the same movement of the control column as above. $110 \mathrm{kt}$ is the cruising airspeed of DA-40, and $60 \mathrm{kt}$ is the airspeed, at which the aeroplane is following landing procedures. During landing, the control column is involved in many parts of the procedures.

The total force on the control column without the aerodynamic force generated 


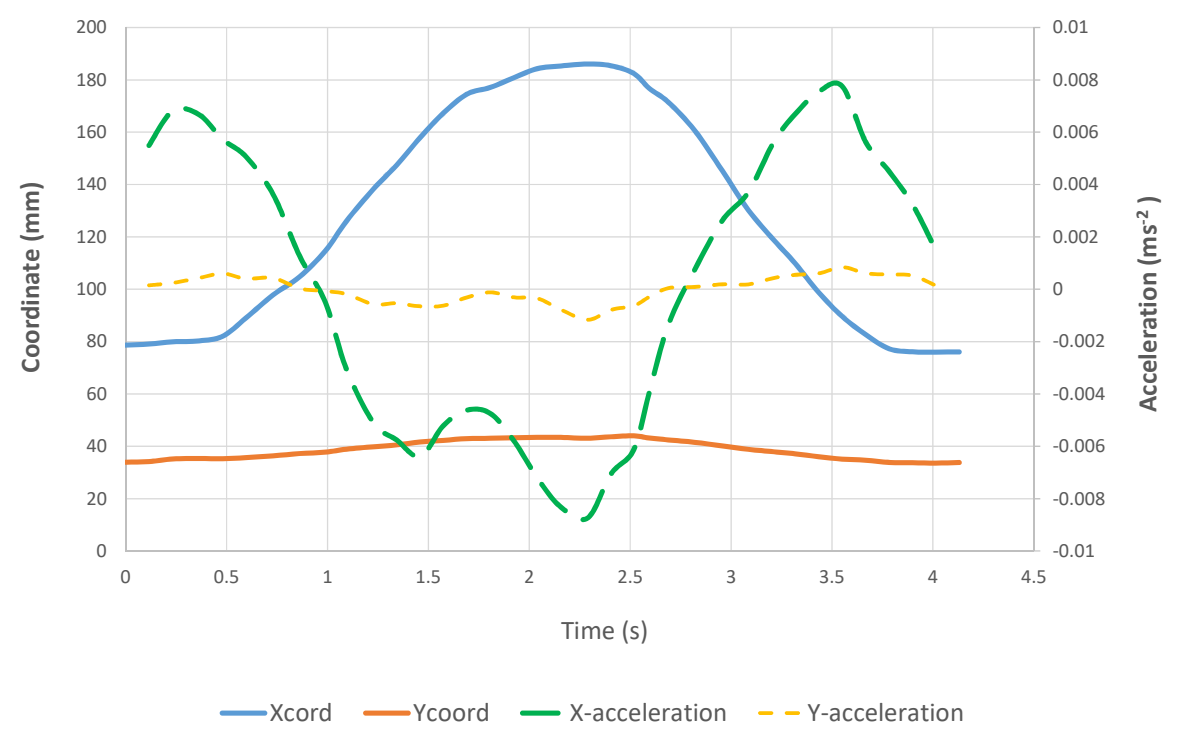

Figure 7: Control column's movement

by ailerons (the system force) and the total force on the control, when the airspeed is $60 \mathrm{kt}$, and $110 \mathrm{kt}$, are calculated. The comparison of the forces on the control on ground and in flight is shown in Figure 9 for $60 \mathrm{kt}$, and in Figure 10 for $110 \mathrm{kt}$.

It can be seen that the aerodynamic component of the force on the control becomes dominant when it is in flight. The percentage of the system force in the total force on the control is about $15 \%$ at $60 \mathrm{kt}$, and the percentage decreases to approximately $10 \%$ at $110 \mathrm{kt}$.

However, the proportion of the system force is high when the acceleration of the control movement is high, for example, when the time is at about $0.5 \mathrm{~s}$ and about at $3.5 \mathrm{~s}$.

The profile of the force on the control column in flight is approximately proportional to that of the displacement, which is similar to that of F_Sx. 


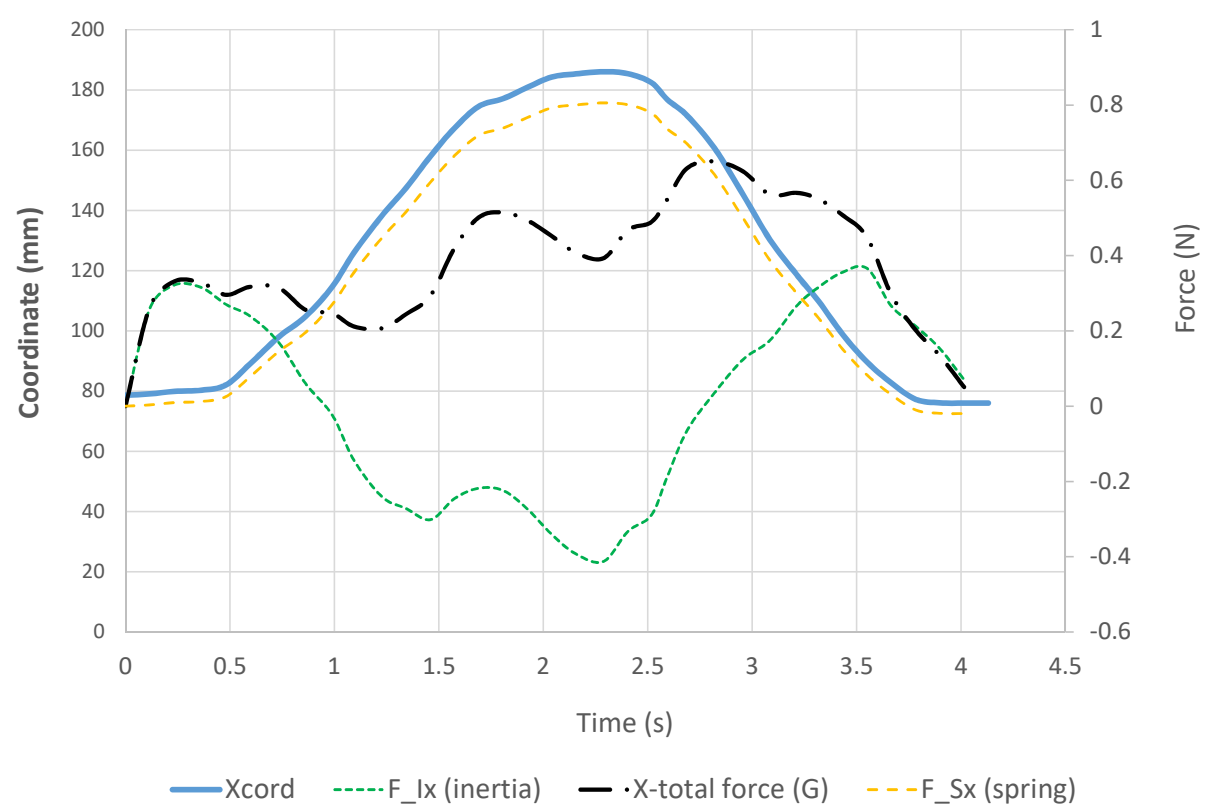

Figure 8: Force on control column on ground

The aerodynamic force generated by a flight control surface is proportional to the deflection angle of the surface, according to the model assumptions, and the the deflection angle changes linearly with the displacement of the control column. Therefore, it performs as a strong "spring". However this "spring constant" is not constant, which will change with airspeed and air density.

The observations from the calculation by the model discussed above need to be repeated, in particular, with the movement of the control column measure in actual flights, and the value of force measurement taken directly from the control column. The measurement of the control's movement in flight has to overcome variation of light intensity in the cockpit. A significant change in light intensity produces interferences to the image processing. The technique to resolve this difficulty and a force sensing system is in development. 


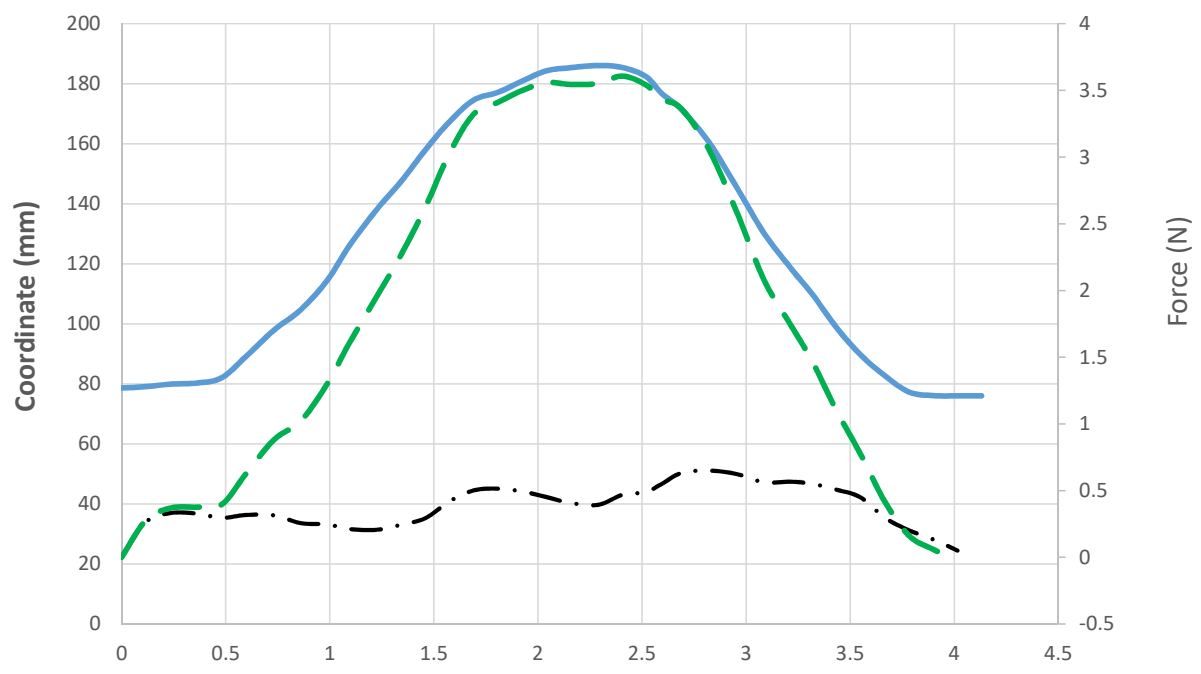

Time (s)

-Xcord $\quad$ - X-total force (G) $\longrightarrow$ X-Total force (60kt)

Figure 9: Force on control column (airspeed $=60 \mathrm{kt}$ )

\section{Conclusion}

The 2-dimensional force model has been used to analyse the forces generated by each element in the linkage systems to flight control surfaces. These forces contribute to the force exerted on the control column in the cockpit. The model forms the basis of force feed-back on the control column of a PCATD simulator. An image sensing system to measure the movement of control column in the cockpit of an aeroplane has been developed. It has been operated under restricted conditions inside the cockpit. The calculations of the force on control have been carried out by the force model with the input of the series of the control's coordinates changing with time. The results from the calculation have shown the characteristics of the force on 


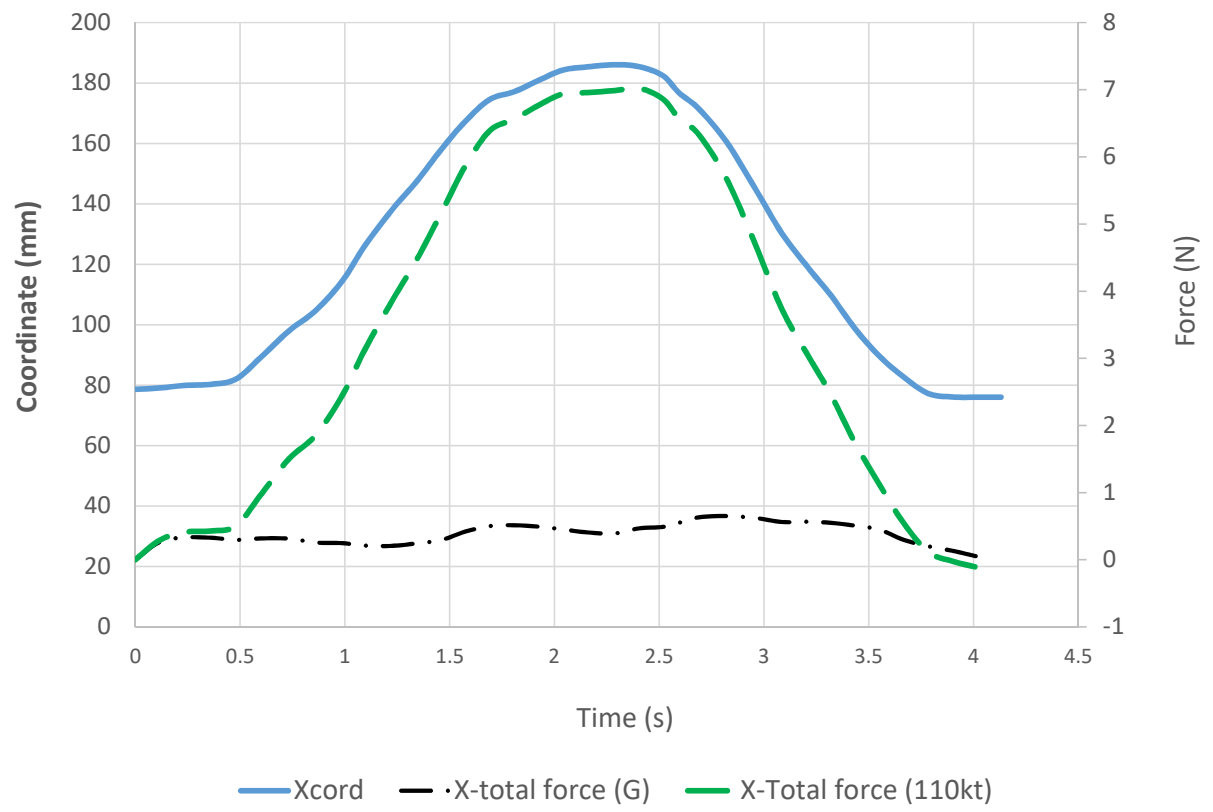

Figure 10: Force on control column (airspeed $=100 \mathrm{kt}$ )

the control column on ground and in flight. The aerodynamic force becomes a much greater contributor to the force on control, in particular, when the acceleration of the control is not high. The proportion of the system force, i.e without aerodynamic force, increases when the airspeed decreases.

The results also show that the assumption that describes the flight control surfaces, elevator and aileron as symmetrical thin aerofoils leads to a simple linear relationship between the lift coefficient of the flight control surfaces and the displacement of control column. This shows that the aerodynamic force change with the displacement of the control column acts like a force caused by a spring.

The information the calculation results have displayed can provide some useful suggestions or strategy for the future implementation of the force feed-back 
onto the PCATD simulator.

The next step of the model development will concentrate on the calculations with the in flight measurement of the control's movement, and the measurement of actual force on the control column on ground and in flight.

Acknowledgments We appreciate the help of the flight instructors, Akanksha Sharma, Aeron Warner and the aircraft engineers at the Milson Flight System Centre of the School of Aviation, and the effort of Fraser Johnson, 4th year Engineering student, to make the measurements involved in this paper possible.

\section{References}

[1] Edward Lewis Houghton and Peter William Carpenter. Aerodynamics for engineering students. Elsevier, 2003. C238

[2] AC Revised Kermode, edited by RH Barnard, and DR Philpott. Mechanics of flight, 1996. C238

[3] Savern L Reweti, Andrew Gilbey, and Lynn Jeffrey. Efficacy of low-cost PC-based aviation training devices. Journal of Information Technology Education-Research, 16:127-142, 2017. C234

[4] Yoonsu Nam. Qft force loop design for the aerodynamic load simulator. IEEE Transactions on Aerospace and Electronic Systems, 37(4):1384-1392, 2001, doi:10.1109/7.976973. C233

[5] Garrett Weinberg and Bret Harsham. Developing a low-cost driving simulator for the evaluation of in-vehicle technologies. In Proceedings of the 1st International Conference on Automotive User Interfaces and Interactive Vehicular Applications, pages 51-54. ACM, 2009, doi:10.1145/1620509.1620519. C234 


\section{Author addresses}

1. R G Davies, School of Aviation, Massey University, Palmerston North 4442, New ZeAland.

mailto:R.G.Davies@massey . ac .nz

2. G Sen Gupta, School of Engineering and Advanced Technology, Massey University, Palmerston North 4442, NEW ZEALAND. 